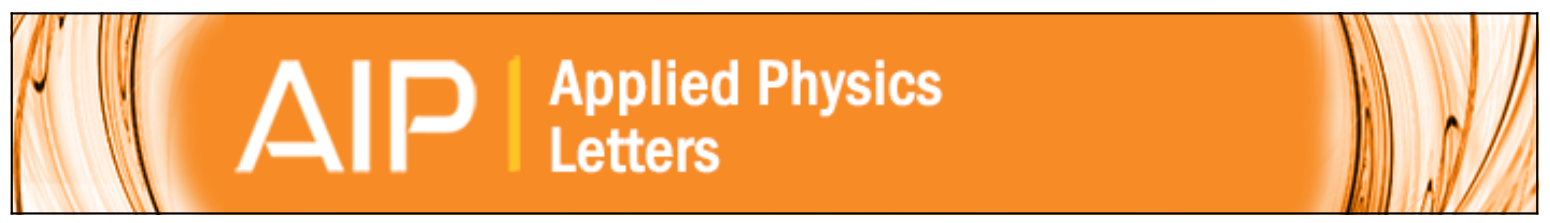

\title{
Enhanced Raman scattering from vertical silicon nanowires array
}

Jian-An Huang, Ying-Qi Zhao, Xue-Jin Zhang, Lin-Bao Luo, Yan-Kuan Liu, Juan Antonio Zapien, Charles Surya, and Shuit-Tong Lee

Citation: Applied Physics Letters 98, 183108 (2011); doi: 10.1063/1.3584871

View online: http://dx.doi.org/10.1063/1.3584871

View Table of Contents: http://scitation.aip.org/content/aip/journal/apl/98/18?ver=pdfcov

Published by the AIP Publishing

\section{Articles you may be interested in}

Diameter dependence of polarization resolved reflectance from vertical silicon nanowire arrays: Evidence of tunable absorption

J. Appl. Phys. 114, 024304 (2013); 10.1063/1.4813081

Highly enhanced Raman scattering from coupled vertical silicon nanowire arrays

Appl. Phys. Lett. 101, 173114 (2012); 10.1063/1.4764057

The growth and radial analysis of $\mathrm{Si} / \mathrm{Ge}$ core-shell nanowires

Appl. Phys. Lett. 97, 251912 (2010); 10.1063/1.3531631

Enhanced field emission from $\mathrm{ZnO}$ nanowires grown on a silicon nanoporous pillar array

J. Appl. Phys. 108, 114301 (2010); 10.1063/1.3516156

The effect of plasmonic particles on solar absorption in vertically aligned silicon nanowire arrays Appl. Phys. Lett. 97, 071110 (2010); 10.1063/1.3475484

\section{A|P| Journal of}

Journal of Applied Physics is pleased to announce André Anders as its new Editor-in-Chief 


\title{
Enhanced Raman scattering from vertical silicon nanowires array
}

\author{
Jian-An Huang, ${ }^{1}$ Ying-Qi Zhao, ${ }^{2}$ Xue-Jin Zhang, ${ }^{1}$ Lin-Bao Luo, ${ }^{1}$ Yan-Kuan Liu, ${ }^{1}$ \\ Juan Antonio Zapien, ${ }^{1}$ Charles Surya, ${ }^{2}$ and Shuit-Tong Lee ${ }^{1, a)}$ \\ ${ }^{1}$ Department of Physics and Materials Science, Center of Super-Diamond and Advanced Films (COSDAF), \\ City University of Hong Kong, Hong Kong \\ ${ }^{2}$ Department of Electronic and Information Engineering, Hong Kong Polytechnic University, Hong Kong
}

(Received 16 March 2011; accepted 12 April 2011; published online 5 May 2011)

\begin{abstract}
We fabricated ordered hexagonal-packed vertical silicon nanowire (SiNW) arrays with varying diameters of 450-900 $\mathrm{nm}$ and varying lengths of 0.54-7.3 $\mu \mathrm{m}$, and studied their Raman enhancement properties. We found the Raman enhancement per unit volume (REV) increased with decreasing wire diameters and oscillated with wire length, and the REV of seven 450-nm-diameter, $3-\mu \mathrm{m}$-long SiNWs was about twice that of a single SiNW having the same size. The differences were attributed to the vertical finite-length cylinder structures of the SiNW array, as supported by finite-difference-time-domain simulation results based on the helical resonant surface wave model. (C) 2011 American Institute of Physics. [doi:10.1063/1.3584871]
\end{abstract}

Since silicon ( $\mathrm{Si}$ ) is an inefficient light-emitting material, the stimulated Raman amplification of $\mathrm{Si}$ is instead more suitable for realizing lasing. ${ }^{1-4}$ Current Si Raman lasers could indeed be achieved with a lasing threshold of $20 \mathrm{~mW}$ via a $2 \mathrm{~cm}$ Si ring resonator. ${ }^{5}$ As ultra compact $\mathrm{Si}$ lasers with a lower threshold are needed for photonic applications, submicrometer $\mathrm{Si}$ waveguides have been investigated and shown to have enhanced Raman gain and low loss. ${ }^{6,7}$ Individual $\mathrm{Si}$ nanowire $(\mathrm{SiNW})$ is also a promising laser candidate, which has been shown to exhibit a strong Raman enhancement factor of $\sim 800$ compared to bulk $\mathrm{Si}^{8}{ }^{8}$ However, realization of SiNW Raman laser still faces challenges, such as poor reproducibility in fabrication via bottom-up methods and difficult manipulation of small-sized SiNWs of high Raman gain. In this letter, we present the controlled fabrication of ordered vertical aligned SiNW arrays with tunable diameter and length, and detailed study of their Raman activity. We found Raman enhancement of SiNW arrays was morphology dependent, and about twice that of a single SiNW.

We fabricated ordered SiNW arrays by using nanosphere lithography and metal-induced etching as before ${ }^{9}$ with slight modifications. Briefly, polystyrene (PS) microspheres (microsphere $\mathrm{GmbH}$ ) of $\sim 980 \mathrm{~nm}$ in diameter, were diluted 1:1 by volume ratio with ethanol, and injected into the water surface of a Petri dish containing a p-type $\langle 100\rangle \mathrm{Si}$ wafer and deionized water till all water surface was covered by a PS monolayer. The PS monolayer was lifted up slowly by the $\mathrm{Si}$ wafer underneath, after which the wafer was allowed to dry in air. Reactive ion etching was used to etch the PS using oxygen gas at 40 SCCM (SCCM denotes cubic centimeter per minute at STP) flow rate and 30 mTorr, and the etching time was varied to control the diameters of PS microspheres. The substrate was next coated with a gold layer of $20 \mathrm{~nm}$ by electronic beam deposition, and ultrasonicated in chloroform for $1 \mathrm{~min}$ to remove the PS monolayer. The wafer was then immersed in a mixed solution of $0.4 \mathrm{M}$ hydrofluoric acid and hydrogen peroxide under ambient air condition for 5 to 20 min to obtain SiNWs arrays of different lengths. The asprepared SiNW arrays were washed by aqua regia to remove

${ }^{a)}$ Electronic mail: apannale@cityu.edu.hk. the gold layer, cleaned by deionized water, and dried by nitrogen gas for Raman measurement.

The as-made vertical SiNW arrays shown in Figs. 1(a) and 1(b) were highly ordered and hexagonally packed with uniform diameter and length, which enabled stable and reproducible measurement of Raman scattering. The intensity of the first-order Raman scattering of $\mathrm{Si}\left(520 \mathrm{~cm}^{-1}\right)$ from the SiNWs arrays was compared to that from $\mathrm{Si}$ wafer to obtain the Raman enhancement per unit volume (REV), defined as $\operatorname{REV}=\left(I_{\mathrm{nw}} / V_{\mathrm{nw}}\right) /\left(I_{\text {bulk }} / V_{\text {bulk }}\right),{ }^{8}$ where $I$ is the Raman intensity of SiNW (nw) or Si wafer (bulk) and $V$ the corresponding Raman sampling volume. The measurement was conducted in the backscattering scheme [Fig. 1(c)] with a Renishaw InVia Raman system equipped with a HeNe laser of $632.8 \mathrm{~nm}$ at $17 \mathrm{~mW}$ power and its polarization direction labeled by $E$. As illustrated in Fig. 1(d), the scheme would excite the SiNWs lying within the laser spot of diameter $D$,

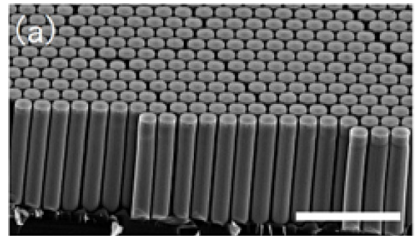

(c)

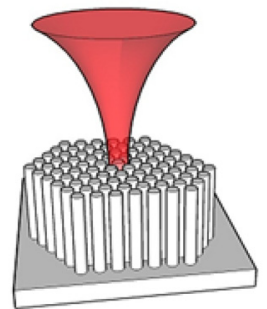

(d)

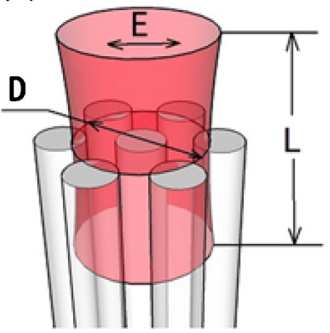

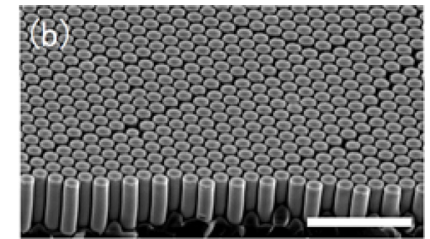

(e)

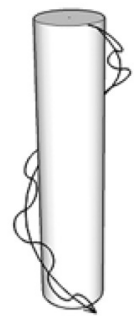

FIG. 1. (Color online) SEM image of SiNWs array of diameter of $850 \mathrm{~nm}$ and length of (a) $\sim 7.30$ and (b) $5.21 \mu \mathrm{m}$. The scale bar is $5 \mu \mathrm{m}$. (c) Illustration of backscattering scheme of Raman measurement. The excitation laser is focused by an objective onto the SiNW array. Backscattering means that the scattered Raman signals of the SiNW array are collected by the objective in the reverse path of the excitation laser. (d) Sampling volume of diameter $D$ and height $L$, and $E$ the polarization direction. (e) Illustration of a helical resonant surface wave on the surface of a finite cylinder. 

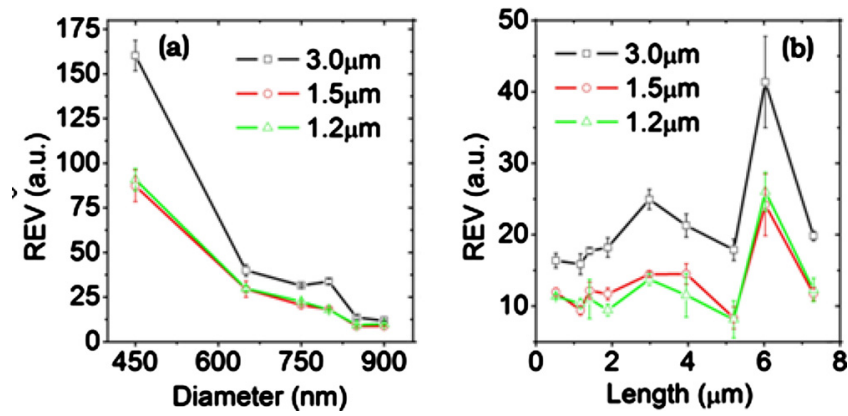

FIG. 2. (Color online) (a) Change in REV vs wire diameters of SiNW array of $\sim 3.0 \mu \mathrm{m}$ wire length under different laser spot sizes. (b) REV change vs wire lengths of SiNW array of $850 \mathrm{~nm}$ in diameter under different laser spot sizes.

and collect the Raman signals emitted primarily from the SiNWs enclosed within a cylindrical sampling volume of diameter $D$ and height $L,{ }^{10}$ defined theoretically as $L$ $=4 \lambda /\left\{\pi \tan ^{2}\left[\sin ^{-1}\right.\right.$ (N.A.) $\left.]\right\}$, where $\lambda$ is the wavelength of the excitation laser, and N.A. the numerical aperture of the objective used. To investigate the dependence of REV on wire diameter and length, we prepared SiNW arrays with varying diameters of 450-900 nm, and varying lengths of $0.54-7.3 \mu \mathrm{m}$. All SiNW arrays had periodicity of $\sim 1 \mu \mathrm{m}$ as they were prepared with the same PS microsphere template. Three objectives with a laser spot size of $\sim 3.0 \mu \mathrm{m}$, $1.5 \mu \mathrm{m}$, and $1.2 \mu \mathrm{m}$, respectively, were used to probe the Raman signals of one or more SiNWs to study the REV difference.

The changes in REV with the wire diameter of SiNW arrays and laser spot sizes are shown in Fig. 2(a) with the length of the SiNW arrays fixed at $\sim 3.00 \mu \mathrm{m}$. It shows that REV increases with decreasing wire diameter and becomes larger as more SiNWs are probed by a larger laser spot. For the laser spots of $\sim 1.2 \mu \mathrm{m}$ and $1.5 \mu \mathrm{m}$ that would detect, respectively, one and two SiNWs on average, the REV curve shows little difference, whereas the REV clearly becomes larger for the $\sim 3.0 \mu \mathrm{m}$ laser spot that would probe seven SiNWs. Significantly, for the SiNWs of $450 \mathrm{~nm}$ in diameter the REV excited by the $\sim 3.0 \mu \mathrm{m}$ laser spot is $\sim 160$, while the REV excited by the $\sim 1.2 \mu \mathrm{m}$ spot is only 90.7 , thus the former REV is almost twice as large as the latter. Figure 2(b) shows all three REV curves for SiNWs of $850 \mathrm{~nm}$ in diameter oscillate with wire length and the REV excited by the $\sim 3.0 \mu \mathrm{m}$ laser spot is distinctly larger than of the two smaller laser spots.

The Raman signal of SiNW array arises from the inelastic scattering of the incident light by the optical phonon in the $\mathrm{Si}$ crystal, whereas Raman enhancement is due to morphology-dependent resonance of the Raman signal in the SiNW array. ${ }^{11,12}$ Cao et al. ${ }^{8}$ demonstrated the resonance of Raman signal in single SiNW by calculating an elastic scattering model of infinite-length dielectric cylinder. The model explained the increasing REV with decreasing SiNW diameters as in Fig. 2(a), although it could not account for the effect of wire length. Deeper understanding of the resonance in dielectric cylinders and spheres revealed the presence of surface-waves that encircled the excited object and interfered constructively with one another to give the resonance. ${ }^{12-17} \mathrm{In}$ our case of finite-length SiNW array, the surface-waves interpretation should also be able to explain the REV variation with wire length and laser spot size. To check this supposi-
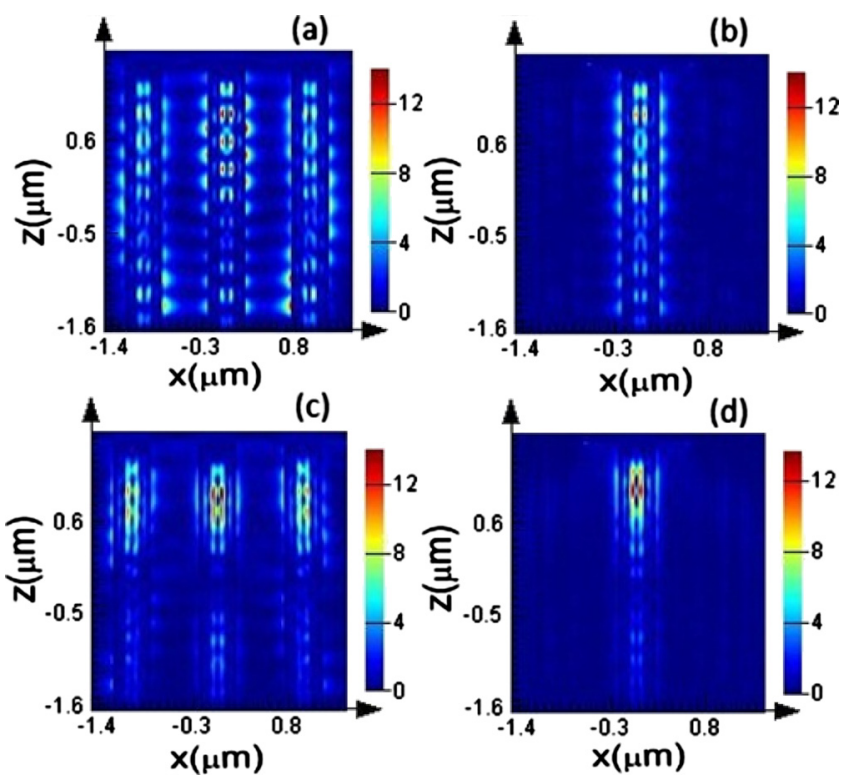

FIG. 3. (Color online) FDTD-simulated cross-sectional distributions of normalized electrical intensity $\left(I_{E} / I_{0}\right)$ of three SiNWs of $450 \mathrm{~nm}$ in diameters and $3.0 \mu \mathrm{m}$ in length under excitation of (a) $\sim 3.0$ - $\mu \mathrm{m}$-width laser of 654 $\mathrm{nm}$, (b) $\sim 1.2$ - $\mu \mathrm{m}$-width laser of $654 \mathrm{~nm}$, (c) $\sim 3.0$ - $\mu$ m-width laser of 633 $\mathrm{nm}$, and $(\mathrm{d}) \sim 1.2-\mu \mathrm{m}$-width laser of $633 \mathrm{~nm}$. The laser polarization direction is along the $\mathrm{x}$ axis.

tion, we used finite-difference-time-domain (FDTD) (Refs. 18-20) method to simulate SiNW array resonances under different laser spots by calculating the elastic scattering of linearly polarized monochromatic incident plane waves by vertical Si cylinders in vacuum as in Fig. 1(d). As the enhancement process involved the incident light and the Raman light, two plane waves of the original wavelength $\boldsymbol{\lambda}_{0}$ $=633 \mathrm{~nm}$ and Raman-shifted wavelength $\boldsymbol{\lambda}_{1}=654 \mathrm{~nm}$, respectively, were used to evaluate the Raman enhancement using $Q_{\text {Raman }}=Q_{\text {incident }}\left(\boldsymbol{\lambda}_{\mathbf{0}}\right) Q_{\text {scatter }}\left(\boldsymbol{\lambda}_{\mathbf{1}}\right),{ }^{8}$ where $Q=I_{E} / I_{0}$ is the normalized electric intensity of SiNW.

Figure 3(a) shows the cross-sectional distribution of the normalized electrical intensities on three SiNWs of the seven SiNWs excited by the $\sim 3.0-\mu \mathrm{m}$-wide laser of $654 \mathrm{~nm}$, revealing that electrical standing waves of different shapes appear on the surface of seven SiNWs, whereas Fig. 3(b) shows that weaker waves appear only on the middle SiNW as it is the sole wire excited by the $1.2-\mu \mathrm{m}$-wide laser of the same wavelength. These standing waves are the resonant surface waves that helically propagate on the surface of the finitelength cylinder as illustrated in Fig. 1(e). ${ }^{16,17}$ Figures 3(c) and 3(d) show similar results under $633 \mathrm{~nm}$ excitation but most resonant electrical intensities are confined inside the SiNWs. From $Q_{\text {Raman }}=Q_{\text {incident }}\left(\boldsymbol{\lambda}_{\mathbf{0}}\right) Q_{\text {scatter }}\left(\boldsymbol{\lambda}_{\mathbf{1}}\right)$, the enhancement should be contributed by resonances of both the incident light and the Raman light. The incident light is first scattered by the SiNW into all directions. The azimuthal component of the light scattered into the SiNW is totally reflected by the curved inner wall of the SiNW to form a wire diameter-dependent head-to-tail light path that matches the constructive interference conditions to establish resonance inside the SiNW. This internal resonant electric field interacts inelastically with the SiNW crystal to emit the Raman light. The Raman light is then scattered again by the SiNW to build resonances not only inside the SiNW but also on its surface The resonant helical surface wave appears 

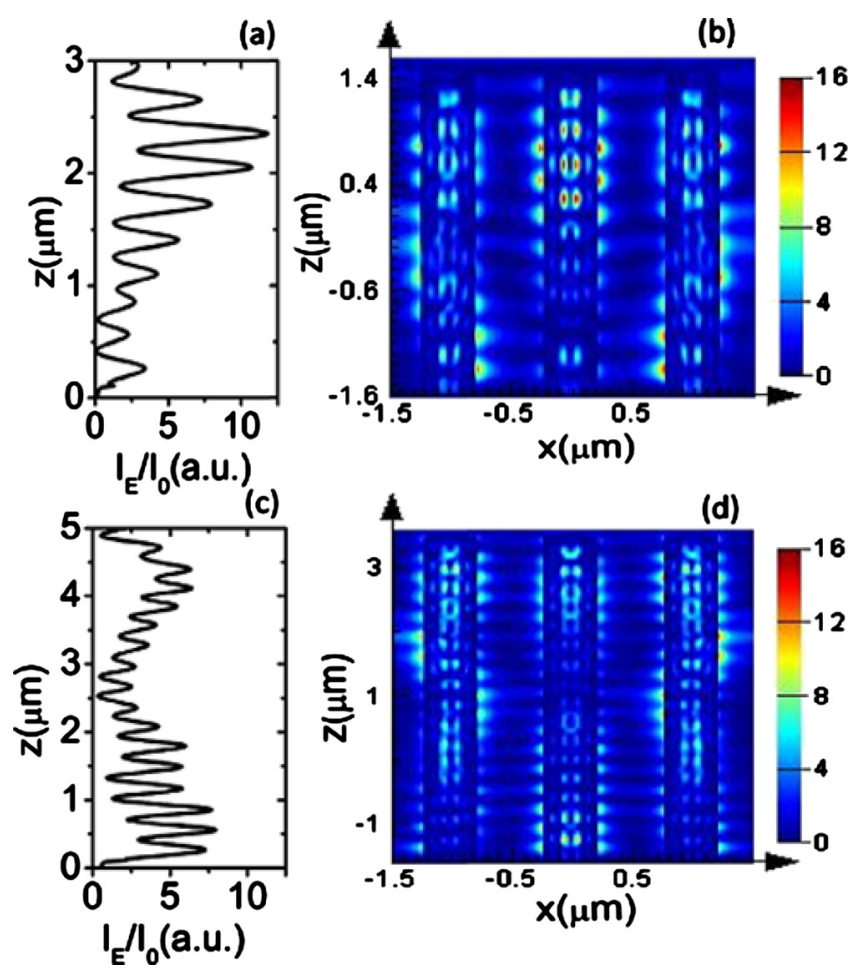

FIG. 4. (Color online) FDTD-stimulated profiles of normalized electrical intensity $\left(I_{E} / I_{0}\right)$ along the axial direction ( $\mathrm{z}$ axis) on the surfaces of the middle SiNW of $850 \mathrm{~nm}$ in diameter and lengths of (a) $3 \mu \mathrm{m}$, (c) $5 \mu \mathrm{m}$, respectively, under $\sim 3.0-\mu \mathrm{m}$-width laser of $654 \mathrm{~nm}$, (b) the corresponding cross-sectional electric distribution of SiNWs in (a), and (d) the corresponding cross-sectional electric distribution of SiNWs in (c).

because as the wavelength of the Raman light increases $\left(\boldsymbol{\lambda}_{1}=654 \mathrm{~nm}\right)$, the resonant azimuthal component inside the wire leaks out on the SiNW surface, and the axial component on the SiNW surface is reflected by the base of the SiNW to form a wire length-dependent head-to-tail path and satisfy the resonance condition. Although the REV already removes the contribution of internal field resonance of the incident light, the surface wave resonance of the Raman light leads to the increase in REV with the number of excited SiNWs because the total electrical intensities on the surface waves of seven SiNWs in Fig. 3(a) is apparently larger than that of the single SiNW in Fig. 3(b).

On the other hand, there are many helical surface wave propagating back and forth along the SiNW due to the base reflection, and their constructive interference occurs only on specific resonant diameter and length of the SiNW. ${ }^{17}$ From the resonance of infinite-length cylinder in the work of Cao et al. ${ }^{8}$ we can see that the SiNW diameter dominates the resonance. Under the resonant diameter, it is the finite length of the SiNW that gives rise to a discrete set of pitch angles of the helical surface waves. ${ }^{17}$ Thus different wire lengths cor- respond to different surface resonance patterns (Fig. 4), and the varied total surface electrical intensities result in the REV oscillation with wire length.

In conclusion, ordered SiNWs arrays with controllable sizes were fabricated. Raman enhancement from SiNWs arrays was found to increase with decreasing wire diameter, to oscillate with wire length, and to increase with increasing number of SiNWs probed. The variation was due to the morphology-dependent resonance of SiNWs, and could be explained by an elastic scattering model of helical resonant surface waves and supported by FDTD simulation. The present understanding of the enhancement mechanism would benefit the design and development of Si nanophotonic devices as well as Si Raman laser.

This work was supported by the Research Grants Council of Hong Kong SAR, China (Grant Nos. CityU5/CRF/08, CityU 101807, CityU 103409, and PolyU 5302/09E).

${ }^{1}$ R. Claps, D. Dimitropoulos, V. Raghunathan, Y. Han, and B. Jalali, Opt. Express 11, 1731 (2003).

${ }^{2}$ O. Boyraz and B. Jalali, Opt. Express 12, 5269 (2004).

${ }^{3}$ H. S. Rong, R. Jones, A. S. Liu, O. Cohen, D. Hak, A. Fang, and M. Paniccia, Nature (London) 433, 725 (2005).

${ }^{4}$ H. S. Rong, A. S. Liu, R. Jones, O. Cohen, D. Hak, R. Nicolaescu, A. Fang, and M. Paniccia, Nature (London) 433, 292 (2005).

${ }^{5}$ H. S. Rong, S. B. Xu, Y. H. Kuo, V. Sih, O. Cohen, O. Raday, and M. Paniccia, Nat. Photonics 1, 232 (2007).

${ }^{6}$ T. J. Kippenberg, S. M. Spillane, D. K. Armani, and K. J. Vahala, Opt. Lett. 29, 1224 (2004).

${ }^{7}$ Q. F. Xu, V. R. Almeida, and M. Lipson, Opt. Lett. 30, 35 (2005).

${ }^{8}$ L. Y. Cao, B. Nabet, and J. E. Spanier, Phys. Rev. Lett. 96, 157402 (2006).

${ }^{9}$ K. Q. Peng, M. L. Zhang, A. J. Lu, N. B. Wong, R. Q. Zhang, and S. T. Lee, Appl. Phys. Lett. 90, 163123 (2007).

${ }^{10}$ E. Smith and G. Dent, Modern Raman Spectroscopy: A Practical Approach (Wiley, Hoboken, NJ, 2005).

${ }^{11}$ J. F. Owen, R. K. Chang, and P. W. Barber, Aerosol Sci. Technol. 1, 293 (1982).

${ }^{12}$ P. W. Barber and R. K. Chang, Optical Effects Associated with Small Particles (World Scientific, Singapore/Teaneck, NJ, USA, 1988).

${ }^{13}$ H. Inada and M. A. Plonus, IEEE Trans. Antennas Propag. AP18, 649 (1970).

${ }^{14}$ H. Inada and M. A. Plonus, IEEE Trans. Antennas Propag. AP18, 89 (1970).

${ }^{15}$ J. D. Murphy, P. J. Moser, A. Nagl, and H. Uberall, IEEE Trans. Antennas Propag. 28, 924 (1980).

${ }^{16}$ A. Nagl, H. Uberall, P. P. Delsanto, J. D. Alemar, and E. Rosario, Wave Motion 5, 235 (1983).

${ }^{17}$ H. Überall, P. J. Moser, B. L. Merchant, A. Nagl, K. B. Yoo, S. H. Brown, J. W. Dickey, and J. M. D'Archangelo, J. Appl. Phys. 58, 2109 (1985).

${ }^{18}$ M. I. Mishchenko, J. W. Hovenier, and L. D. Travis, Light Scattering by Nonspherical Particles:Theory, Measurements, and Applications (Academic Press, San Diego, 2000).

${ }^{19}$ A. Taflove and S. C. Hagness, Computational Electrodynamics: The Finite-Difference Time-Domain Method (Artech House, Boston, 2005).

${ }^{20}$ FDTD Solution, www.lumerical.com. 